\title{
Fanconi Anemia: Hematopoietic Stem Cell Transplant or Gene Therapy?
}

\author{
Parisa Naji ${ }^{1}$, Maryam Behfar ${ }^{1}$, Arefeh Jafarian ${ }^{1}$, and Amir Ali Hamidieh ${ }^{2}$ \\ ${ }^{1}$ Tehran University of Medical Sciences \\ ${ }^{2}$ Pediatric Cell Therapy Research Center, Tehran University of Medical Sciences
}

December 15, 2021

\begin{abstract}
FA is a rare, multi-organ cancer-prone IBMFS associated with hematological malignancies and STs. The androgen therapy, hematopoietic growth factors, HSCT, and GT, still in the clinical trial, are various treatments for this disease. Here, we aimed to compare the advantages and disadvantages of HSCT and GT in FA cures. We perform an advanced electronic search of "FA" AND (genetics OR treatment OR HSCT OR GT OR Mosaicism), and "Allo-HSCT" AND (conditioning regimen OR complications OR GvHD OR infection OR cost) MeSH terms in non-citation and citation databases. Besides, the gray literature was searched too. This article will provide a summary of the advantages and disadvantages of HSCT and GT of FA disease. Our results show that GT has a good potential in FA treatments in the future. Furthermore, it has higher advantages and fewer disadvantages in comparison with HSCT. Systematic Review Registration: CRD42021247364 ID on PROSPERO database.
\end{abstract}

\section{Title Page}

Fanconi Anemia: Hematopoietic Stem Cell Transplant or Gene Therapy?

\section{Authors}

Parisa Naji, MSc ${ }^{1}$, Maryam Behfar, Dr ${ }^{1,2}$, Arefeh Jafarian, Dr ${ }^{3}$, Amir Ali Hamidieh, Prof ${ }^{1 *}$

${ }^{1}$ Pediatric Cell and Gene Therapy Research Center, Tehran University of Medical Sciences, Tehran, Iran

${ }^{2}$ Pediatric Hematopoietic Stem Cell Transplant Department, Children's Medical Center, Tehran University of Medical Sciences, Tehran, Iran

${ }^{3}$ Iranian Tissue Bank Research Center, Tehran University of Medical Sciences, Tehran, Iran

\section{* Correspondence to:}

Amir Ali Hamidieh, Professor, Pediatric Cell and Gene Therapy Research Center, Tehran University of Medical Sciences, Tehran, Iran, Children's Medical Center, Qarib St, Keshavarz Blvd, Tehran 14194, Iran, Phone: +98 216147 2911, Fax: +98 218353 2375, E-mail: aahamidieh@tums.ac.ir

Main Text Word Count: 3484

Abstract Word Count: 147

Short Running Title: FA: HSCT or GT?

Keywords: Fanconi Anemia, Hematopoietic Stem Cell Transplantation, Gene Therapy, Comparison.

Tables: 3

Figures: 1 
Abbreviations

\begin{tabular}{ll}
\hline FA & Fanconi Anemia \\
IBMF & Inherited Bone Marrow Failure \\
ICL & Inter-strand DNA Cross-Links \\
rhIL-3 & Interleukin 3 \\
rhGM-CSF & Granulocyte-Macrophage Colony-Stimulating Factor \\
rhG-CSF & Granulocyte Colony-Stimulating Factor \\
HSCT & Hematopoietic Stem Cell Transplantation \\
GT & Gene Therapy \\
Allo-HSCT & Allogeneic Hematopoietic Stem Cell Transplantation \\
GvHD & Graft versus Host Disease \\
MSD & Matched Sibling Donor \\
OS & Overall Survival \\
MUD & Mismatched Unrelated Donor \\
CMV & Cytomegalovirus \\
AD & Alternative Donor \\
Haplo-HSCT & Haploidentical Hematopoietic Stem Cell Transplantation \\
TCD & T-Cell Depleted \\
ATG & Anti-Thymocyte Globulin \\
CY & Cyclophosphamide \\
SAA & Severe Aplastic Anemia \\
TAI & Thoraco-Abdominal Irradiation \\
FLU & Fludarabine \\
QoF & Quality of Life \\
OM & Oral Mucositis \\
HNSCC & Head and Neck Squamous Cell Carcinomas \\
HC & Hemorrhagic Cystitis \\
PRES & Posterior Reversible Encephalopathy Syndrome \\
CsA & Cyclosporine A \\
TTP & Thrombotic Thrombocytopenic Purpura \\
AML & Acute Myeloid Leukemia \\
ST & Solid Tumor \\
TRM & Transplantation Related Mortality \\
PID & Primary Immunodeficiency Disorder \\
SCID & Severe Combined Immunodeficiency \\
HSPC & Hematopoietic Stem Cell/Progenitor Cells \\
iPSC & Induced Pluripotent Stem Cells \\
\hline &
\end{tabular}

\begin{abstract}
Fanconi anemia (FA) is a rare, multi-organ cancer-prone inherited bone marrow failure syndrome (IBMFS) associated with hematological malignancies and solid tumors. The androgen therapy, hematopoietic growth factors, hematopoietic stem cell transplantation (HSCT), and gene therapy (GT), still in the clinical trial, are various treatments for this disease. Here, we aimed to compare the advantages and disadvantages of HSCT and GT in FA cures.

We perform an advanced electronic search of "Fanconi anemia" AND (genetics OR treatment OR HSCT OR GT OR Mosaicism), and "Allo-HSCT" AND (conditioning regimen OR complications OR GvHD OR infection OR cost) MeSH terms in non-citation and citation databases. Besides, the gray literature was searched too. This article will provide a summary of the advantages and disadvantages of HSCT and GT of
\end{abstract}


FA disease.

Our results show that GT has a good potential in FA treatments in the future. Furthermore, it has higher advantages and fewer disadvantages in comparison with HSCT.

Systematic Review Registration: CRD42021247364 ID on PROSPERO database.

Keywords: Fanconi Anemia, Hematopoietic Stem Cell Transplantation, Gene Therapy, Comparison. 